University of Montana

ScholarWorks at University of Montana

7-2009

\title{
Ontogeny and Individual Variation in the Adrenocortical Response of Zebra Finch (Taeniopygia Guttata) Nestlings
}

Haruka Wada

Katrina G. Salvante

Emily Wagner

Tony D. Williams

Creagh W. Breuner

University of Montana - Missoula, creagh.breuner@umontana.edu

Follow this and additional works at: https://scholarworks.umt.edu/biosci_pubs

Part of the Biology Commons

Let us know how access to this document benefits you.

\section{Recommended Citation}

Wada, Haruka; Salvante, Katrina G.; Wagner, Emily; Williams, Tony D.; and Breuner, Creagh W., "Ontogeny and Individual Variation in the Adrenocortical Response of Zebra Finch (Taeniopygia Guttata) Nestlings" (2009). Biological Sciences Faculty Publications. 73.

https://scholarworks.umt.edu/biosci_pubs/73

This Article is brought to you for free and open access by the Biological Sciences at ScholarWorks at University of Montana. It has been accepted for inclusion in Biological Sciences Faculty Publications by an authorized administrator of ScholarWorks at University of Montana. For more information, please contact

scholarworks@mso.umt.edu. 


\section{Ontogeny and Individual Variation in the Adrenocortical Response of Zebra Finch (Taeniopygia guttata) Nestlings}

\author{
Haruka Wada, ${ }^{1, *}$ \\ Katrina G. Salvante ${ }^{2}$ \\ Emily Wagner ${ }^{3}$ \\ Tony D. Williams ${ }^{3}$ \\ Creagh W. Breuner ${ }^{4}$ \\ ${ }^{1}$ Department of Fisheries and Wildlife Sciences, Virginia \\ Polytechnic Institute and State University, 100 Cheatham \\ Hall, Blacksburg, Virginia 24061; ${ }^{2}$ Department of Biology, \\ University of North Carolina, Chapel Hill, North Carolina \\ 27599; ${ }^{3}$ Department of Biological Sciences, Simon Fraser \\ University, Burnaby, British Columbia V5A 1S6, Canada; \\ ${ }^{4}$ Division of Biological Sciences, University of Montana, \\ Missoula, Montana 59812
}

Accepted 9/10/2008; Electronically Published 5/8/2009

\begin{abstract}
Numerous studies indicate interspecies variation in the ontogeny of the adrenocortical response in birds; however, little is known about the extent of interindividual variation in avian young. Toward this end, we examined the ontogeny and interindividual variation in the adrenocortical response in zebra finch (Taeniopygia guttata) nestlings. We measured baseline and stress-induced total (bound and free) corticosterone, corticosteroid binding globulin capacity, and resulting estimated free corticosterone levels in nestlings of four different ages (days 5 , 10,16 , and 21). In addition, we investigated the potential correlates of interindividual variation (brood size and mass). Nestlings at days 5 and 10 post-hatching showed no significant increase in total or free corticosterone levels in response to a standardized handling stress, whereas an adult-like stress response was seen by day 16 post-hatching. There was large interindividual (fivefold) variation in both baseline and stressinduced corticosterone among individual nestlings at any age. We estimate that half of this individual variation in the adrenocortical response could be explained by between-clutch variation (e.g., genetics), while the other half could be explained by other factors such as rearing environment (based on estimated intraclass correlation coefficients). Total baseline corticosterone, but not stress-induced corticosterone, was negatively correlated with fledging mass in this species.
\end{abstract}

* Corresponding author; e-mail: haruka@vt.edu.

Physiological and Biochemical Zoology 82(4):325-331. 2009. (C) 2009 by The University of Chicago. All rights reserved. 1522-2152/2009/8204-8165\$15.00 DOI: $10.1086 / 599320$

\section{Introduction}

Glucocorticoids and catecholamines are two of the main mediators of allostasis (i.e., organisms' constant alterations in physiology to predictable and unpredictable events) and are secreted according to the allostatic state of the individual (McEwen and Wingfield 2003). Glucocorticoids, in particular, modify energy allocation to increase chances of survival, for example, to escape from environmental challenges. At the same time, prolonged, excessive levels of these hormones bear detrimental consequences, especially for developing animals, as they can divert resources away from growth (Morici et al. 1997; Glennemeier and Denver 2002), alter cognition (Kitaysky et al. 2003; but see also Catalani et al. 2000), and modify hypothalamic-pituitary-adrenal (HPA) axis reactivity (Catalani et al. 2000; Hayward and Wingfield 2004). Thus, it has been hypothesized that HPA axis reactivity should be dampened during a rapid-growth phase in developing animals (Sapolsky and Meaney 1986; Walker et al. 2001). This stress-hyporesponsive period (SHRP) may be a result of any of the following: decreased hormone secretion at any level of the HPA axis, elevated plasma corticosteroid binding globulin (CBG) levels, decreased sensitivity at the target tissue, or enhanced sensitivity to increased negative feedback at the neural level.

Ontogeny of the adrenocortical response in birds appears to depend on functional maturity at hatching (precocial vs. altricial, i.e., lack of down/feathers and locomotor activity at hatching). Depending on where a species falls in the precocialaltricial spectrum, mobility and dependency on parents for food and brooding differ (Starck and Ricklefs 1998). As a consequence, young birds vary in the ability to escape environmental or predatory challenges, and thus they vary in how much they can benefit from secreting glucocorticoids in response to such challenges. While more independent and mobile precocial young may benefit from elevating corticosterone (CORT) levels in response to a food shortage, nest-bound altricial young may suffer from deleterious effects of the hormone (the developmental hypothesis; Schwabl 1999; Sims and Holberton 2000; Kitaysky et al. 2003; Blas et al. 2006; Wada et al. 2007). Thus, the hypothesis predicts that precocial species will develop an adult-like adrenocortical response earlier than nonprecocial species, and the latter group will establish this response when the young animals can benefit from glucocorticoid secretion, for example, when they become mobile and more independent.

Timing in the establishment of the HPA reactivity in various bird species follows the prediction based on the developmental 
hypothesis. For instance, precocial chickens and turkeys show an adrenocortical response to heat stress before hatching (Jacobs 1996). However, heat and/or cold stress does not elicit a response again until $2 \mathrm{~d}$ post-hatch, indicating that the SHRP lasts for $\sim 48$ h (Freeman and Flack 1980; Freeman 1982; Freeman and Manning 1984). Among a couple of semialtricial species studied, nestlings show a gradual increase in the magnitude of the adrenocortical response during the nestling period (Love et al. 2003; Walker et al. 2005a). In contrast to altricial birds, Magellanic penguin (Spheniscus magellanicus) hatchlings show an adult-like response to adrenocorticotropic hormone challenge (Walker et al. 2005a) and 10-d-old kestrel nestlings (Falco sparverius; out of 28-d nestling period) can significantly elevate CORT levels in response to capture and handling (Sockman and Schwabl 2001; Love et al. 2003). In altricial birds, there is a suggestion of an early hyporesponsive period post-hatching, but the data are inconclusive due to a lack of experiments on prehatching individuals (Wada et al. 2007). In Passeriformes, northern mockingbirds (Mimus polyglottos) do not respond to handling stress until they are near independence (Sims and Holberton 2000), while white-crowned sparrows (Zonotrichia leucophrys nuttalli) show a robust response before fledging (Wada et al. 2007). A similar pattern to the one observed in temperate white-crowned sparrows is observed in white storks (Ciconiiformes: Ciconia ciconia; Blas et al. 2005, 2006). Therefore the relative timing in the maturation of the HPA axis is not consistent among altricial species; however, all altricial species studied to date respond to stress later than precocial species, as predicted by the hypothesis. It is important to point out that we cannot dismiss the possibility that altricial species simply have a delayed maturation of the HPA axis without an adaptive function. It is possible that altricial and precocial young follow the same postfertilization development, with a difference in when hatching occurs, and the HPA axis maturity in altricial young corresponds to that of precocial embryos. In order to determine whether the dampened HPA reactivity in altricial birds is adaptive, true existence of the SHRP needs to be examined.

The above-mentioned studies suggest substantial interspecies variation in ontogeny of the adrenocortical response in birds; however, little is known about the extent of intraspecific or interindividual variation. In mammals, individual variation in adult HPA axis reactivity is attributed largely to postnatal environment (Caldji et al. 2001). Early experiences such as handling and maternal separation can permanently alter sensitivity of the HPA axis. For nonmammalian taxa, far less is known about individual variation in endocrine systems. In adults, individual variation in HPA axis reactivity is shown to be repeatable within a breeding season (Cockrem and Silverin 2002) and heritable across generations (Satterlee and Johnson 1988; Tanck et al. 2001; Evans et al. 2006). Recent studies also indicate that postnatal environment, such as exposure to human disturbance, can contribute to the variation in the HPA axis of avian young (Mullner et al. 2004; Walker et al. 2005b). Yet, the existence of the individual variation in nonmammalian young is not well understood; therefore, it is not clear when this variation appears (but see Blas et al. 2007).

In this study, we examined the ontogeny and individual variation in the adrenocortical response in altricial zebra finch (Taeniopygia guttata) nestlings. Specifically, we explored (1) the age-specific effects of standardized handling stress on total CORT, CBG capacity, and resulting estimated free CORT levels; (2) interindividual variation in these endocrine traits at any given age; and (3) correlates of interindividual variation such as brood size and mass. The results of this study can help elucidate novel mechanisms that may contribute to hyporeactivity during critical developmental periods and factors affecting individual variation in young.

\section{Material and Methods}

\section{Study Species and Breeding Protocol}

This experiment was conducted in December 2002, using a captive zebra finch colony at Simon Fraser University, Burnaby, British Columbia, Canada. Zebra finches are an altricial species: nestlings hatch with their eyes closed and with only a few downy feathers (Zann 1996). Nestlings begin to open their eyes by day 6 or 7 , and eyes are fully open by day 10. Thermoregulatory ability is mostly completed by day 4 or 5 , pinfeathers break through the skin around day 6, and fledging takes place at approximately $18 \mathrm{~d}$ of age. However, young birds do not reach adult mass until $51 \mathrm{~d}$ of age.

All the birds in this experiment were maintained in controlled conditions: $19^{\circ}-23^{\circ} \mathrm{C}$ temperature, $14 \mathrm{~L}: 10 \mathrm{D}$ photoperiod, and 35\%-55\% humidity. Adults were provided with mixed seeds (1:3 Panicum and white millet, $11.7 \%$ protein, $0.6 \%$ lipid, and $84.3 \%$ carbohydrate by dry mass), water, grit, and cuttlefish bone ad lib. In addition, a multivitamin supplement was added to the drinking water once a week. Breeding pairs also received an egg food supplement (20.3\% protein, $6.6 \%$ lipid) between pairing and clutch completion. The pairs were housed in separate cages with an external nest box (15 $\mathrm{cm} \times 14.5 \mathrm{~cm} \times 20 \mathrm{~cm})$. Nest boxes were checked daily between 0900 and 1100 hours for new eggs and hatchlings, and laying and hatching orders were recorded. When nestlings hatched, they were banded on day 10 . Brood size at the time of blood sampling varied between two and six chicks. The protocol used in this experiment was approved by the Canadian Committee on Animal Care (CCAC) and the University of Texas Institutional Animal Care and Use Committees (IACUC).

\section{Measuring Adrenocortical Responses}

To evaluate the HPA reactivity of zebra finch nestlings, we used capture and handling stress as a standardized stressor (Wingfield 1994). This standardized procedure allows comparison of the HPA reactivity between ages and species. Nestlings were sampled at four ages: approximately day 5, 10, and 16 nestlings and approximately day 21 fledglings. A total of 22 nests ( $n=6,6,6$, and 4 for 5-, 10-, 16-, and 21-d ages, respectively) were sampled, and each nest was sampled for one age group 


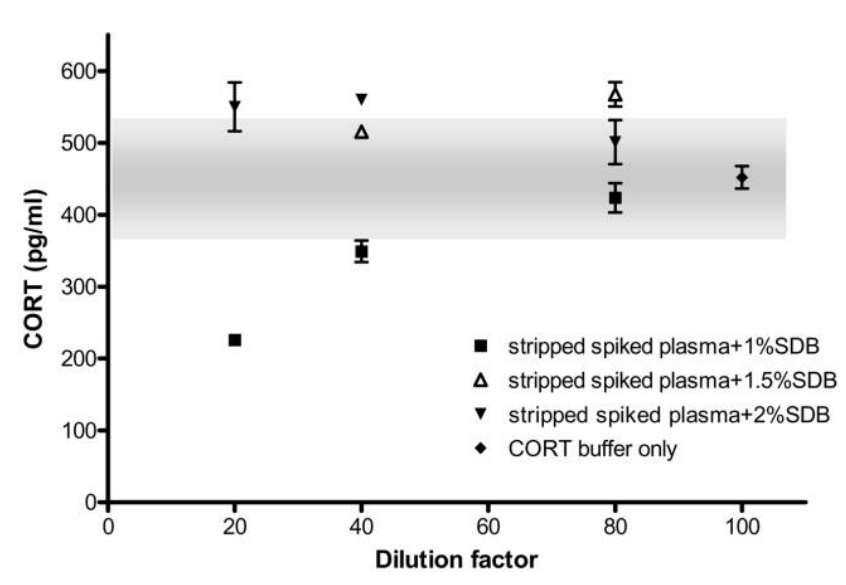

Figure 1. Optimization of enzyme immunoassay corticosterone (CORT) assay for zebra finch plasma. Plasma was stripped and spiked to create $500 \mathrm{pg} \mathrm{mL}^{-1}$ CORT, then it was diluted with a $500 \mathrm{pg} \mathrm{mL}^{-1}$ CORT buffer for each concentration of the steroid displacement buffer (SDB). As plasma dilution factor increased, the value became closer to the added concentration of CORT, indicating the appropriate SDB concentrations for the given dilution factor with a negligible interference between plasma and the assay. Additional optimization with $1.5 \%$ SDB indicated that a dilution factor of $1: 40$ with $1.5 \%$ SDB eliminated any measurable effects of plasma in the assay. Values at $1.5 \%$ SDB (from the latter assay) were corrected for interassay variation on this plot. Gray box indicates the mean \pm SEM of the CORT buffer with which the samples were diluted.

only to avoid habituation to handling. To avoid collecting an excessive amount of blood from the young (5-d-old nestlings weigh an average of $5.4 \mathrm{~g}$ ), we divided the nestlings from one nest into two groups: nonstress baseline and restraint-induced stress groups. All the nestlings from each nest were captured at the same time. Half of the nestlings were bled within $3 \mathrm{~min}$ of opening the nest box (baseline); the other half were bled after $30 \mathrm{~min}$ of restraint in an opaque cloth bag. For blood sampling, the alar vein was punctured with a 26-gauge needle, and the welling blood was collected with heparinized capillary tubes (approximately $30 \mu \mathrm{L}$ ). Samples were centrifuged for 5 min at 2,200 $g$ within a few hours of collection; plasma was removed and stored at $-20^{\circ} \mathrm{C}$. Plasma samples were then shipped on dry ice to the University of Texas at Austin, where all the assays were performed.

\section{Enzyme Immunoassay for CORT}

We used enzyme immunoassay kits from Assign Designs (Ann Arbor, MI) to determine plasma CORT levels (see Wada et al. 2007 for details). We first optimized the assay for zebra finch plasma by testing various plasma dilutions $(1: 10,1: 20,1: 40$, $1: 60$, and $1: 90$ ) and steroid displacement buffer (SDB; to inhibit CORT from binding to CBG or other proteins) concentrations $(0 \%, 1 \%, 1.5 \%$, and $2 \%)$. Over several assays, we determined the optimum to be 1:40 plasma dilutions with 1.5\% SDB (Fig. 1).

For this study, we ran samples in triplicate, with 1: 40 plasma dilution and $1.5 \%$ SDB. Intra- and interassay variations were
$7.4 \%$ and $15.6 \%$, respectively. Detectability for the assay was

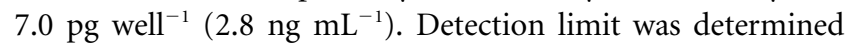
by taking 2 standard deviations from the mean of the blank wells. Detection limit of the plate was assigned to undetectable samples in the assay.

\section{Radio-Ligand Binding Assay for CBG}

Radio-ligand binding assays were used to measure the CBG levels in zebra finch nestling plasma. Zebra finch CBG was first characterized in plasma dilution and equilibrium binding assays using pooled plasma. Then CBG levels in individual samples were measured in the point sample assay. In all assays, plasma was stripped to remove endogenous CORT and then incubated with either ${ }^{3 \mathrm{H}} \mathrm{CORT}$ and buffer (total binding) or ${ }^{3 \mathrm{H}} \mathrm{CORT}$ and cold CORT (nonspecific binding). Bound and free ${ }^{3 \mathrm{H}} \mathrm{CORT}$ were separated by vacuum filtration over a glass filter, and levels of bound ${ }^{3 \mathrm{H}} \mathrm{CORT}$ were assessed with a scintillation counter. The optimal dilution, $1: 1,620$, and 2 -h incubation at $4^{\circ} \mathrm{C}$ were used in all assays. The intraassay variation was $3.8 \%$.

In order to estimate free CORT levels, we used an equation by Barsano and Baumann (1989):

$$
\begin{aligned}
H_{\text {free }}= & 0.5 \times\left[H_{\text {total }}-B_{\text {max }}-1 / K_{\mathrm{a}}\right. \\
& \left. \pm \sqrt{\left(B_{\max }-H_{\text {total }}+1 / K_{\mathrm{a}}\right)^{2}+4\left(H_{\text {total }} / K_{\mathrm{a}}\right)}\right]
\end{aligned}
$$

where $K_{\mathrm{a}}$ is $1 / K_{\mathrm{d}}$ (nM; $K_{\mathrm{d}}$ is affinity of CORT for CBG [determined in the equilibrium binding assay]), $B_{\max }$ is total CBG capacity, and $H_{\text {total }}$ is total plasma hormone concentration.

\section{Data Analysis}

All statistical analyses were performed using SPSS 12.0. The effects of handling and age on total and free CORT and CBG levels were determined using one-way repeated-measures ANOVA followed by Tukey HSD, with age as a main factor. Pairwise comparisons were done using Bonferroni corrections. Between- and within-clutch variability in total CORT was examined using intraclass correlation analysis with a two-way random effect model ( $r=$ [between-groups variance - withingroups variance]/[between-groups variance + within-groups variance]). To explore factors influencing individual variation in hormone levels, baseline and stress-induced levels were analyzed separately. Using hierarchical linear regression analyses, three correlates of interindividual variation were regressed against baseline and stress-induced total and free CORT levels: brood size, individual mass at the time of sampling, and mass at fledging (approximately day 21). Individual mass at the time of sampling depended on the sampling age; thus, unstandardized residuals in linear regression were used to disregard age. Levene's test was used to test heteroscedasticity of CORT and CBG levels, and values were log transformed when $P$ was less than 0.05 (total and free CORT). 


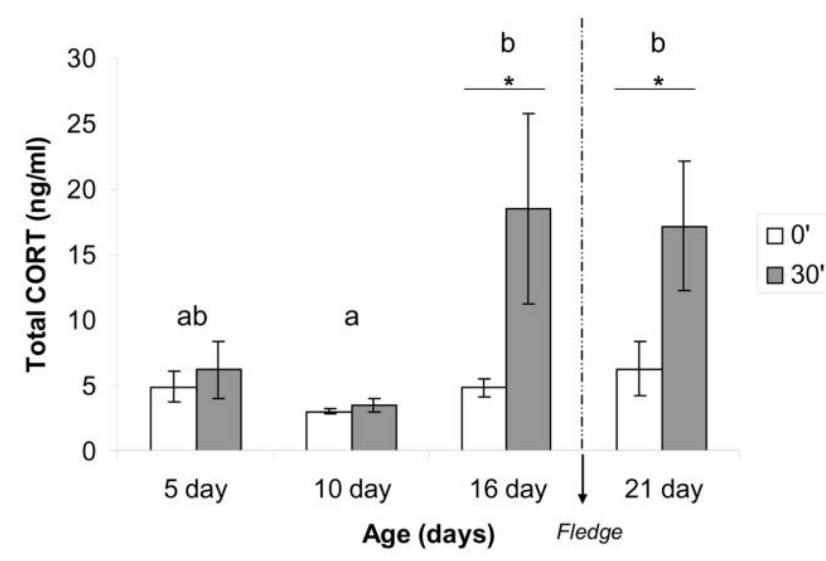

Figure 2. Changes in total corticosterone (CORT) secretion in response to handling stress, by age. An asterisk denotes a significant adrenocortical response within the age group $(P<0.05)$. Different letters indicate an overall age difference in adrenocortical responses $(P<0.05)$. $N=6,6,6$, and 4 for 5-, 10-, 16-, and 21-d ages, respectively.

\section{Results}

Ontogeny of the Adrenocortical Response

Overall, there were significant effects of age $\left(F_{3,18}=8.09\right.$, $P=0.001$, power $=0.971)$ and handling time $\left(F_{1,20}=10.98\right.$, $P=0.004$, power $=0.879)$ and a marginally significant interaction between age and time $\left(F_{3,18}=2.82, P=0.068\right.$, power $=0.577$ ) on total (bound and unbound) plasma CORT levels (Fig. 2). Post hoc tests for age comparison show that day 10 nestlings had significantly lower responses than day 16 and day 21 nestlings, while responses of day 5 nestlings were not significantly different from any other age groups $(P>0.05)$. Day 16 and day 21 were the only age groups that showed a significant CORT increase during restraint $(P<0.05)$. In addition, an average coefficient of variation for baseline and stressinduced total CORT was $50.2 \%, 25.3 \%, 66.5 \%$, and $54.6 \%$ for day $5,10,16$, and 21 nestlings, respectively (excluding samples below the detection limit).

For CBGs, the equilibrium saturation binding assay estimated CORT affinity for nestling CBG $\left(K_{\mathrm{d}}\right)$ at $5.13 \pm 0.62 \mathrm{nM}$ (Fig. 3 ). This nestling affinity estimate was used to calculate free CORT levels in this study. Overall, there were no significant effects of age or handling time on CBG capacity (age: $F_{3,18}=1.56, P=$ 0.233 , power $=0.341$; time: $F_{1,20}=0.34, P=0.569$, power $=$ $0.085)$. Similarly, there was no significant interaction between time and age $\left(F_{3,18}=0.76, P=0.529\right.$, power $\left.=0.181\right)$.

Since we detected no effect of age on CBG during nestling development, the pattern of free (unbound) CORT was parallel to that of total CORT. Overall there were significant effects of age $\left(F_{3,18}=4.34, P=0.018\right.$, power $\left.=0.782\right)$ and handling time $\left(F_{1,20}=12.58, P=0.002\right.$, power $\left.=0.918\right)$, and there was a marginal interaction between age and time $\left(F_{3,18}=2.57, P=\right.$ 0.086 , power $=0.535$ ) on free CORT levels (Fig. 4 ). Post hoc tests for age comparisons showed that day 10 nestlings had significantly lower responses than day 16 nestlings $(P<0.05)$.

\section{Correlates of Interindividual Variation in HPA Reactivity}

There was large interindividual variation in both baseline and stress-induced CORT (Fig. 5). In order to quantify the extent of variation explained by clutch versus other factors, we used intraclass correlation coefficients (ICCs). The ICC is a measure of homogeneity of variances within clutches compared with the total. The ICCs for 0 - and 30-min total CORT were 0.467 and 0.577 , respectively. Positive values indicate that between-clutch variation is larger than within-clutch variation. Values of approximately 0.5 indicate that half of observed variation is explained by between-clutch variation (parents' genetics or degree of parental care), while the other half is explained by other factors.

While neither mass nor brood size correlated with total stress-induced levels, fledging mass (mass at approximately day 21) correlated significantly and negatively with total baseline CORT ( $r^{2}=0.19, P=0.042$; Fig. 6 ). Adding mass or brood size at the time of sampling did not significantly increase this $r^{2}$ value in the model (increases in $r^{2}$ value for two factors were 0.006 [ $P=0.705]$ and 0.007 [ $P=0.697]$, respectively). We did not observe any significant correlation between those factors and free CORT levels $(P>0.05)$.

\section{Discussion}

\section{Ontogeny of the Adrenocortical Response in Zebra Finches}

The developmental data in this article indicated no detectable adrenocortical response during the early post-hatch period in zebra finches, at least in individuals up to age $10 \mathrm{~d}$. By day 16 post-hatch, however, nestlings responded to stress with a significant elevation of both total and free CORT; the magnitude of this response is typical of mature, adult birds (Wada et al. 2008). Mean CORT levels were lowest at day 10. These results differ from previous studies investigating the existence of SHRP in altricial birds because previous studies observed a gradual

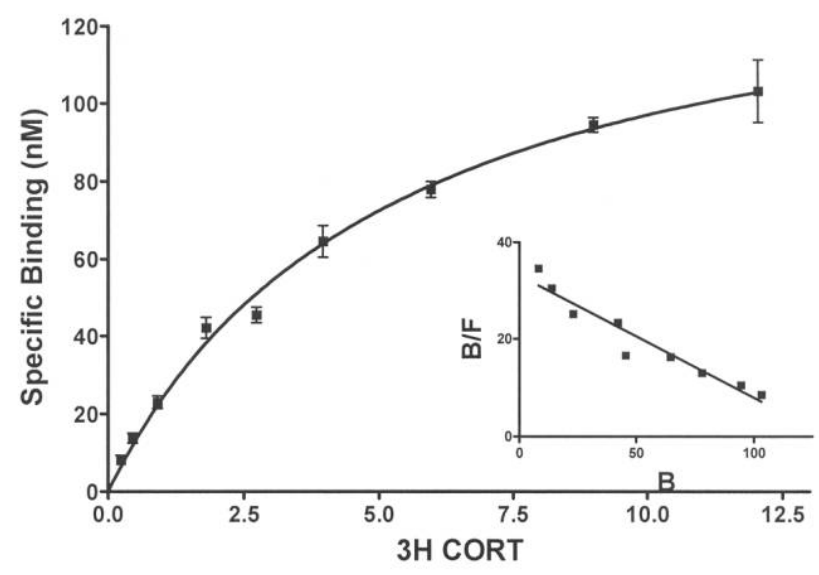

Figure 3. Equilibrium saturation binding of nestling corticosteroid (CORT) binding globulin. Data shown are average specific binding $\pm \mathrm{SE}$ at each concentration and are best fit by a one-site model. Inset is the Scatchard-Rosenthal replot of the data. 


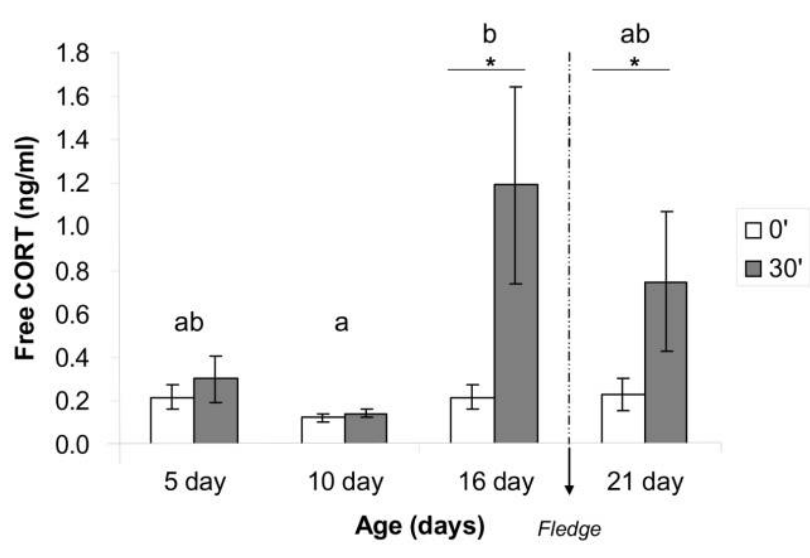

Figure 4. Age-specific adrenocortical responses in free corticosterone (CORT). An asterisk denotes a significant adrenocortical response within the age group $(P<0.05)$. Different letters indicate an overall age difference in adrenocortical responses $(P<0.05) . N=6,6,6$, and 4 for 5-, 10-, 16-, and 21-d ages, respectively.

increase in the response with age (Sims and Holberton 2000; Blas et al. 2006; Wada et al. 2007). Most mammals and precocial birds show a SHRP during development (Freeman and Flack 1980; Freeman 1982; Freeman and Manning 1984; Sapolsky and Meaney 1986; Walker et al. 2001). Implicit in the name is the indication that hormone levels will go down at some time during development when the animal is hyposensitive to stress. As discussed above, this decline in stress reactivity has not yet been detected in altricial birds. Zebra finches, however, showed a trend of reduced stress reactivity on day 10 of nestling development. Nestlings' responses on day 10 appear to be lower than those on day 5 and are significantly lower than those on days 16 and 21. Unfortunately, we did not have enough statistical power to show this potential decline from days 5 to 10 ; more studies with larger sample sizes are needed to confirm this trend.

The CBG pattern observed in this study differed from previous studies. An age-specific increase in CBG is seen in numerous mammals and in birds (D'Agostino and Henning 1981; Viau et al. 1996; Wada et al. 2007). This change in CBG maintains low free CORT levels because (1) an increase in CBG precedes (Tinnikov 1993) or occurs in the absence of (Heo et al. 2003) a rise in total CORT or (2) CBG and CORT secretions rise in parallel (Wada et al. 2007). Thus, a change in CBG level is proposed to be one of the protective mechanisms against deleterious effects of CORT during development (Wada et al. 2007). In contrast, we did not observe an increase in CBG with age in zebra finch nestlings. Thus, age-specific patterns in total and free CORT levels were nearly identical. This indicates that CBG is not a mechanism to keep free CORT levels low in this species.

\section{Individual Variation in Nestlings' Adrenocortical Responses}

Cockrem and Silverin (2002) reported individual variation in adrenocortical response in adult birds; however, very little is known about the presence of such variation in young birds. In our study, individual variation was observed both in baseline and stress-induced total CORT (also see Wada et al. 2008), although the variation in day 10 nestlings was less than half of that in other age groups. This variation in the HPA axis reactivity may be due to genetics, environment, and/or interaction between the two (Ellis et al. 2006). Unfortunately, the sample size was too small to analyze for sex differences or hatching order. Between- and within-clutch comparisons suggested that clutch variation in genetics and parental care, such as brooding and feeding patterns, only explained approximately half of the variation observed in total CORT. This indicates that other environmental conditions, such as body condition and sibling competition, contribute to approximately half of the variation in nestlings' adrenocortical responses.

Among the environmental conditions, body condition/mass appeared to play a role in shaping individual variation in zebra finch nestlings. While brood size or mass at the time of sampling did not correlate with either the total or the free CORT levels, fledging mass correlated negatively with baseline levels. This is consistent with previous studies where CORT levels are

(a) Baseline (0 min) total CORT

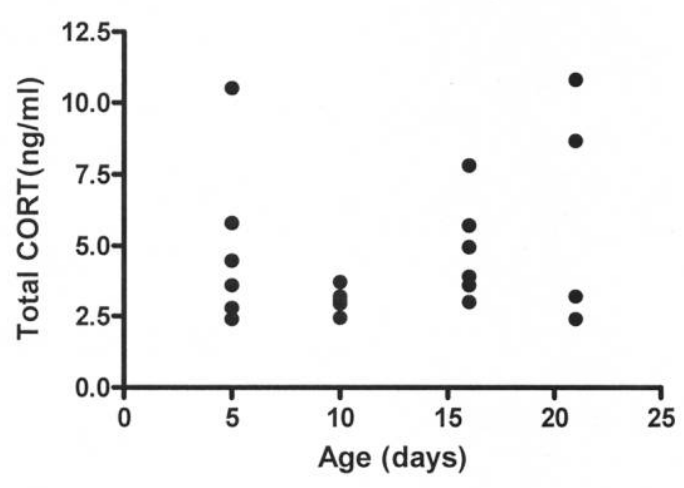

(b) Stressed level $(30 \mathrm{~min})$ total CORT

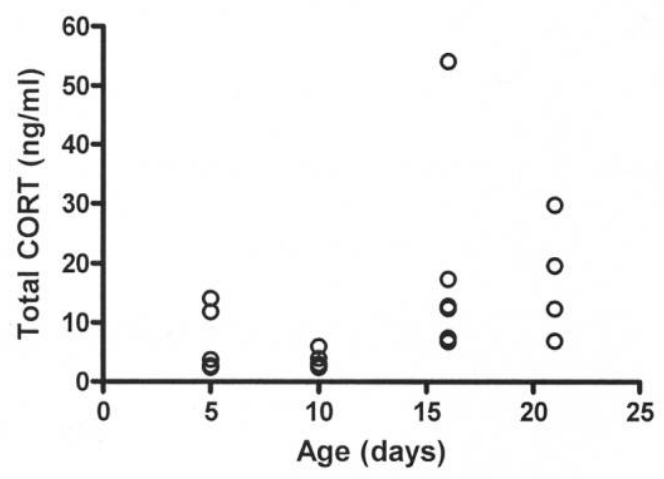

Figure 5. Individual variation in $(a)$ baseline and $(b)$ stress-induced levels of total corticosterone (CORT). $N=6,6,6$, and 4 for 5-, 10-, $16-$, and $21-\mathrm{d}$ ages, respectively. 


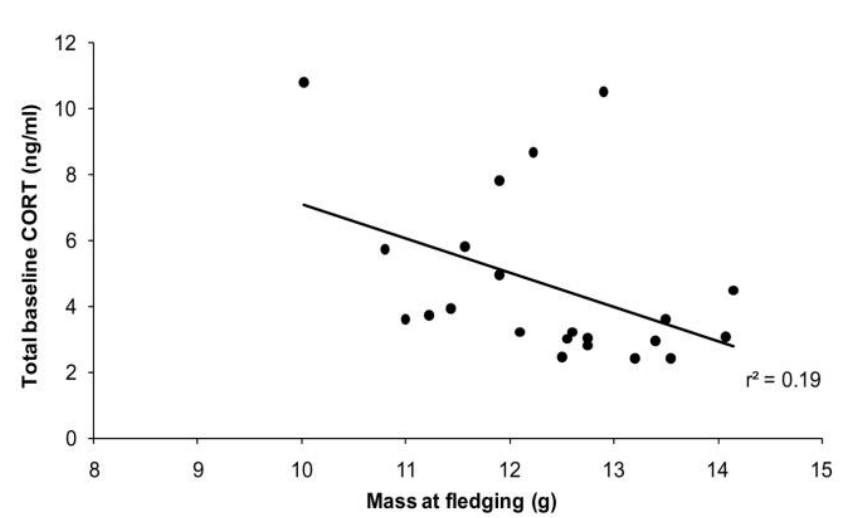

Figure 6. Correlation between mass at fledging (approximately day 21) and total baseline corticosterone (CORT) levels. Regression analysis showed a significant relationship between the two $\left(r^{2}=0.19, P=\right.$ 0.042).

inversely correlated with body condition/mass in birds (Kitaysky et al. 1999a, 1999b; Breuner and Hahn 2003; Love et al. 2005; Wada et al. 2008; but see also Walker et al. 2005a). In adults and some nestlings, increased CORT in smaller, poorcondition birds may increase foraging activity and food intake, which may then lead to a better body condition. At the same time, even moderate levels of CORT treatment are known to retard growth in passerine and amphibian species (Glennemeier and Denver 2002; Wada and Breuner 2008). If CORT retards growth or alters food intake in this species, interindividual variation in CORT and body condition/mass could have longlasting consequences. Further studies are needed to explore the consequences and adaptive significance of this individual variation.

\section{Acknowledgments}

We would like to thank G. Salze and A. Class for their editorial assistance, as well as our funding sources, a Grants-in-Aid-ofResearch award from the Society for Integrative and Comparative Biology (to H.W.), a National Science Foundation grant IBN-0236536 (to C.W.B.), and a Natural Sciences and Engineering Research Council Discovery Grant (to T.D.W.).

\section{Literature Cited}

Barsano C.P. and G. Baumann. 1989. Simple algebraic and graphic methods for the apportionment of hormone (and receptor) into bound and free fractions in binding equilibria; or how to calculate bound and free hormone? Endocrinology 124:1101-1106.

Blas J., R. Baos, G.R. Bortolotti, T. Marchant, and F. Hiraldo. 2005. A multi-tier approach to identifying environmental stress in altricial nestling birds. Funct Ecol 19:315-322.

- 2006. Age-related variation in the adrenocortical response to stress in nestling white storks (Ciconia ciconia) supports the developmental hypothesis. Gen Comp Endocrinol 148:172-180.

Blas J., G.R. Bortolotti, J.L. Tella, R. Baos, and T.A. Marchant. 2007. Stress response during development predicts fitness in a wild, long lived vertebrate. Proc Natl Acad Sci USA 104: 8880-8884.

Breuner C.W. and T.P. Hahn. 2003. Integrating stress physiology, environmental change, and behavior in free-living sparrows. Horm Behav 43:115-123.

Caldji C., D. Liu, S. Sharma, J. Diorio, D. Francis, M.J. Meaney, and P.M. Plotsky. 2001. Development of individual differences in behavioral and endocrine responses to stress: role of the postnatal environment. Pp. 271-292 in B.S. McEwen, ed. Coping with the Environment: Neural and Endocrine Mechanisms. Vol. 4 of The Endocrine System, Sec. 7 of Handbook of Physiology. Oxford University Press, New York. Catalani A., P. Casolini, S. Scaccianoce, F.R. Patacchioli, P. Spinozzi, and L. Angelucci. 2000. Maternal corticosterone during lactation permanently affects brain corticosteroid receptors, stress response and behaviour in rat progeny. Neuroscience 100:319-325.

Cockrem J.F. and B. Silverin. 2002. Variation within and between birds in corticosterone responses of great tits (Parus major). Gen Comp Endocrinol 125:197-206.

D'Agostino J. and S.J. Henning. 1981. Hormonal control of postnatal development of corticosteroid-binding globulin. Am J Physiol 240:E402-E406.

Ellis B.J., J.J. Jackson, and W.T. Boyce. 2006. The stress response systems: universality and adaptive individual differences. Dev Rev 26:175-212.

Evans M.R., M.L. Roberts, K.L. Buchanan, and A.R. Goldsmith. 2006. Heritability of corticosterone response and changes in life history traits during selection in the zebra finch. J Evol Biol 19:343-352.

Freeman B.M. 1982. Stress non-responsiveness in the newlyhatched fowl. Comp Biochem Physiol A 72:251-253.

Freeman B.M. and I.H. Flack. 1980. Effects of handling on plasma corticosterone concentrations in the immature domestic fowl. Comp Biochem Physiol A 66:77-81.

Freeman B.M. and A.C.C. Manning. 1984. Re-establishment of the stress response in Gallus domesticus after hatching. Comp Biochem Physiol A 78:267-270.

Glennemeier K.A. and R.J. Denver. 2002. Small changes in whole-body corticosterone content affect larval Rana pipiens fitness components. Gen Comp Endocrinol 127:16-25.

Hayward L.S. and J.C. Wingfield. 2004. Maternal corticosterone is transferred to avian yolk and may alter offspring growth and adult phenotype. Gen Comp Endocrinol 135:365-371.

Heo J., H.G. Kattesh, M.P. Roberts, and J.F. Schneider. 2003. Plasma levels of cortisol and corticosteroid-binding globulin (CBG) and hepatic CBG mRNA expression in pre- and postnatal pigs. Domest Anim Endocrinol 25:263-273.

Jacobs J. 1996. Regulation of Life History Stages within Individuals in Unpredictable Environments. PhD diss. University of Washington, Seattle.

Kitaysky A.S., E.V. Kitaiskaia, J.F. Piatt, and J.C. Wingfield. 2003. 
Benefits and costs of increased levels of corticosterone in seabird chicks. Horm Behav 43:140-149.

Kitaysky A.S., J.F. Piatt, J.C. Wingfield, and M. Romano. 1999a. The adrenocortical stress-response of black-legged kittiwake chicks in relation to dietary restrictions. J Comp Physiol B 169:303-310.

Kitaysky A.S., J.C. Wingfield, and J.F. Piatt. 1999b. Dynamics of food availability, body condition and physiological stress response in breeding black-legged kittiwakes. Funct Ecol 13: 577-584.

Love O.P., D.M. Bird, and L.J. Shutt. 2003. Corticosterone levels during post-natal development in captive American kestrels (Falco sparverius). Gen Comp Endocrinol 130:135-141.

Love O.P., E.H. Chin, K.E. Wynne-Edwards, and T.D. Williams. 2005. Stress hormones: a link between maternal condition and sex-biased reproductive investment. Am Nat 166:751766.

McEwen B.S. and J.C. Wingfield. 2003. The concept of allostasis in biology and biomedicine. Horm Behav 43:2-15.

Morici L.A., R.M. Elsey, and V.A. Lance. 1997. Effect of longterm corticosterone implants on growth and immune function in juvenile alligators, Alligator mississippiensis. J Exp Zool 279:156-162.

Mullner A., K. Eduard Linsenmair, and M. Wikelski. 2004. Exposure to ecotourism reduces survival and affects stress response in hoatzin chicks (Opisthocomus hoazin). Biol Conserv 118:549-558.

Sapolsky R.M. and M.J. Meaney. 1986. Maturation of the adrenocortical stress response: neuroendocrine control mechanisms and the stress hyporesponsive period. Brain Res Rev 11:65-76.

Satterlee D.G. and W.A. Johnson. 1988. Selection of Japanese quail for contrasting blood corticosterone response to immobilization. Poult Sci 67:25-32.

Schwabl H. 1999. Developmental changes and among-sibling variation of corticosterone levels in an altricial avian species. Gen Comp Endocrinol 116:403-408.

Sims C.G. and R.L. Holberton. 2000. Development of the corticosterone stress response in young northern mockingbirds (Mimus polyglottos). Gen Comp Endocrinol 119:193-201.

Sockman K.W. and H. Schwabl. 2001. Plasma corticosterone in nestling American kestrels: effects of age, handling stress, yolk androgens, and body condition. Gen Comp Endocrinol 122:205-212.

Starck J.M. and R.E. Ricklefs. 1998. Avian Growth and Development: Evolution within the Altricial-Precocial Spectrum. Oxford University Press, New York.
Tanck M.W.T., K.-J. Vermeulen, H. Bovenhuis, and H. Komen. 2001. Heredity of stress-related cortisol response in androgenetic common carp (Cyprinus carpio L.). Aquaculture 199: 283-294.

Tinnikov A.A. 1993. On the role of corticosteroid-binding globulin (CBG) in modulating activity of glucocorticoids: development patterns for CBG, corticosterone, and a-fetoprotein levels in the rat serum. Jpn J Physiol 43:247-251.

Viau V., S. Sharma, and M.J. Meaney. 1996. Changes in plasma adrenocorticotropin, corticosterone, corticosteroid-binding globulin, and hippocampal glucocorticoid receptor occupancy/translocation in rat pups in response to stress. J Neuroendocrinol 8:1-8.

Wada H. and C.W. Breuner. 2008. Transient elevation of corticosterone alters begging behavior and growth of whitecrowned sparrow nestlings. J Exp Biol 211:1696-1703.

Wada H., T.P. Hahn, and C.W. Breuner. 2007. Development of stress reactivity in white-crowned sparrow nestlings: total corticosterone response increases with age, while free corticosterone response remains low. Gen Comp Endocrinol 150:405-413.

Wada H., K.G. Salvante, C. Stables, E. Wagner, T.D. Williams, and C.W. Breuner. 2008. Adrenocortical responses in zebra finches (Taeniopygia guttata): individual variation, repeatability, and relationship to phenotypic quality. Horm Behav 53:472-480.

Walker B.G., J.C. Wingfield, and P.D. Boersma. 2005a. Age and food deprivation affects expression of the glucocorticosteroid stress response in Magellanic penguin (Spheniscus magellanicus) chicks. Physiol Biochem Zool 78:78-89.

- 2005b. Physiological and behavioral differences in Magellanic penguin chicks in undisturbed and tourist-visited locations of a colony. Conserv Biol 19:1571-1577.

Walker C.-D., K.J.S. Anand, and P.M. Plotsky. 2001. Development of the hypothalamic-pituitary-adrenal axis and the stress response. Pp. 237-270 in B.S. McEwen, ed. Coping with the Environment: Neural and Endocrine Mechanisms. Vol. 4 of The Endocrine System. Sec. 7 of Handbook of Physiology. Oxford University Press, New York.

Wingfield J.C. 1994. Modulation of the adrenocortical response to stress in birds. Pp. 520-528 in K.G. Davey, R.E. Peter, and S.S. Tobe, eds. Perspectives in Comparative Endocrinology. National Research Council of Canada, Ottawa.

Zann R.A. 1996. The Zebra Finch: A Synthesis of Field and Laboratory Studies. Oxford University Press, New York. 